\title{
SIMULATIONS OF TRANSITION CROSSING IN THE MAIN INJECTOR
}

\author{
C.M. Bhat and J.A. MacLachlan, \\ Fermi National Accelerator Laboratory * \\ P.O. Box 500, Batavia, IL 60510
}

\begin{abstract}
The design goal for the Fermilab Main Injector (FMI) is to accelerate a minimum of $6 \times 10^{10}$ protons per bunch through the transition. We present here the results from simulation studies of the transition crossing in the FMI using the particle tracking code ESME[1].
\end{abstract}

\section{INTRODUCTION}

The Fermilab Main Injector (FMI)[2] that is under construction is intended to be a high intensity $150 \mathrm{GeV}$ proton injector to the Tevatron. The beam in the FMI will be accelerated from 8 $\mathrm{GeV}$ to $150 \mathrm{GeV}$ through a transition energy of $20.48 \mathrm{GeV}$. The longitudinal emittance of the proton beam at injection is about $0.1 \mathrm{eVs}$, and the intensity will be more than $6 \times 10^{10}$ protons per bunch. Maintaining the beam intensity as well as its longitudinal emittance through the acceleration cycle is very important for the FMI operation. In the past, preserving the beam emittance and the intensity through transition crossing in a proton synchrotron has been one of the major problems. A number of techniques have been suggested to cure these problems[3,4]. Two of the suggested techniques viz., a) $\gamma_{t}$-jump scheme[3] and b) focus free transition crossing(FFTC) [4] have been investigated in some detail for proton synchrotron along with with the normal transition phase jump (NTPJ) scheme. Here, the particle tracking code ESME[1] has been used to study the longitudinal beam dynamics of the transition crossing in the FMI for these three different schemes.

The condition of non- adiabaticity[5] exists in a proton synchrotron when,

$$
\frac{\left|\gamma-\gamma_{t}\right|}{\gamma_{t}} \leq\left[\frac{\gamma_{t}\left(e V_{r f} \sin \phi_{s}\right)^{2}}{4 \pi h E_{o} e V_{r f}\left|\cos \phi_{s}\right|}\right]^{1 / 3}
$$

where $\gamma_{t}$ is the relativistic quantity $\gamma$ at transition, $V_{r f}$ is the peak rf voltage at transition, $\phi_{s}$ is the synchronous angle of the beam with the rf wave form, $h$ is the harmonic number of the machine and $E_{0}$ is the rest mass of proton. By assuming that the $\gamma$ is increasing linearly near transition at a rate $\dot{\gamma}$ this expression can be converted to a non-adiabatic time period in the vicinity of the transition time,

$$
T_{n a}= \pm T_{s}\left[\frac{f_{s} E_{o} \gamma_{t}{ }^{4}}{4 \pi h \dot{\gamma} e V_{r f}\left|\cos \phi_{s}\right|}\right]^{1 / 3}
$$

where $f_{s}=1 / \mathrm{T}_{s}$ is the revolution frequency of the synchronous particle. Since all the particles in a bunch do not pass through

* Operated by the Universities Research Association, under contracts with the U.S. Department of Energy the transition at the same time, there will be a non-linear period during which some particles are above the transition energy while others are below it. The non-linear time is given by,

$$
T_{n l}= \pm \gamma_{t}\left[\frac{\beta^{2}+\alpha_{1} / \alpha_{o}+1 / 2}{\dot{\gamma}}\right] \frac{\Delta p}{p}
$$

where $\beta$ is the ratio of particle velocity and the velocity of light. $\alpha_{1}$ is the second order term in the expansion of path length in $\Delta \mathrm{p} / \mathrm{p}$ and $\alpha_{0}=\gamma_{t}^{-2}$. During this time the rf focusing force causes increased momentum spread and a number of different instabilities come into play. Since the non-adiabatic and nonlinear time decrease with increased $\dot{\gamma}$, the simulations have been carried out for two different values of $\dot{\gamma}$ for the FMI operating scenarios.

\section{ESME SIMULATIONS OF TRANSITION CROSSING}

In ESME, the collective behavior of the beam particles is treated using a pair of Hamilton-like difference equations describing synchrotron oscillations in the energy-angle $(\Delta \mathrm{E}, \phi)$ phase space, (where $\Delta \mathrm{E}=\mathrm{E}-\mathrm{E}_{o}$ and $0 \leq \phi \leq 2 \pi$ ). The particles in a bunch are assumed to have an elliptical distribution which is a good representation of the beam bunches coming from the Fermilab Booster. For a cylindrical beam pipe of radius ' $b$ ' and a co-axial beam of radius ' $a$ ', the impedance, $Z_{\omega}$ seen by a single Fourier component of the beam current at a frequency $\omega / 2 \pi$, is,

$$
\frac{Z_{\omega}}{n}=-j \frac{Z_{o} g}{2 \beta \gamma^{2}}+\frac{Z_{W}}{n}+\frac{Z_{\|}(\omega)}{n}
$$

where $\mathrm{Z}_{o}=377 \mathrm{Ohm}$ (Impedance of free space), $\mathrm{Z}_{W}$ is total wall impedance of the beam pipe and the geometry factor $g=$ $1+2 \ln (b / a)$. The average values of ' $a$ ' and ' $b$ ' are listed in Table I. The $\mathrm{Z}_{\|}$is given by,

$$
Z_{\|}(\omega)=\frac{R_{s}}{1+j Q\left(\frac{\omega_{c}}{\omega}-\frac{\omega}{\omega_{c}}\right)}
$$

For quality factor $\mathrm{Q}=1$, Equation 5 represents the broad-band impedance. $R_{s}$ is the strength of the effective shunt impedance. For the FMI we have taken design value $R_{s}=5 \mathrm{Ohm}$ which is almost surely a considerable over estimate with enough safety margin.

The effect of transverse space charge force producing horizontal betatron tune shift is proportional to the particle density distribution in a bunch at a longitudinal position $\phi$. Very close to the transition, $\eta$ goes to zero. Therefore even a very small correction to $\gamma_{t}$ becomes a sensitive parameter to determine the longitudinal beam dynamics. In the present calculations the dispersion of momentum compaction factor was taken into account by expanding, 
Table I

The parameters used for ESME simulations.

\begin{tabular}{|l|r|}
\hline Parameter & Values \\
\hline Mean radius of FMI & $528.3019 \mathrm{~m}$ \\
\hline$\gamma_{t}$ (nominal) & 21.838 \\
\hline$\dot{\gamma}$ at transition & 167 (Slow Ramp) $\mathrm{sec}^{-1}$ \\
& 300 (Fast Ramp) $\mathrm{sec}^{-1}$ \\
\hline$\alpha_{1}$ & 0.002091 \\
\hline Principal rf sys. & $53 \mathrm{MHz}$ \\
& $4 \mathrm{MV}$ (max) \\
\hline $\begin{array}{l}\text { Init. emittance } \\
\text { and Bunch intensity }\end{array}$ & $0.1 \mathrm{and} 0.2 \mathrm{eVs}$ \\
\hline Coup. imp. Z/| $/ \mathrm{n}$ & $6 \times 10^{10}$ \\
\hline Transverse Beam size(a) & $5 \Omega$ \\
\hline Beam pipe Radius (b) & $2.17 \mathrm{GHz}$ cutoff \\
\hline FFTC : & $0.0022(\mathrm{~m})$ \\
Shaping rf for FFTC & $0.03(\mathrm{~m})$ \\
Type of Tran. Crossing & $159 \mathrm{MHz}$ \\
\hline$\gamma_{t}-$ jump : & $280 \mathrm{kV}(\mathrm{max})$ \\
$\Delta \gamma$ & Non-symmetric \\
Type of Tran. Crossing & \\
\hline
\end{tabular}

$$
\alpha_{p} \approx \alpha_{o}+\left(\alpha_{o}+2 \alpha_{1}-\alpha_{o}^{2}\right) \frac{\Delta p}{p}
$$

For the Main Injector we take $\alpha_{1}$ to be 0.002091 . This corresponds to a Johnsen parameter[3] of 0.8. Thus, each particle has its characteristic $\gamma_{t}$ depending on the deviation of its momentum from that of the synchronous particle. Table I lists the parameters used in the present simulation studies. The results of ESME simulations have been displayed in Table II. The FFTC and $\gamma_{t}$-jump scheme prefer symmetric settings for beam emittance larger than $0.2 \mathrm{eVs}$. For smaller emittance beam, where the space charge forces play important role in emittance blow up, the non-symmetric transition crossing is essential. Figure 1 shows a comparison of evolution of $\epsilon_{l}$ for NTPJ ,FFTC and $\gamma_{t}{ }^{-}$ jump schemes in the Main injector for initial longitudinal emittance of $0.1 \mathrm{eVs}$. All these calculations have been performed by incorporating both space charge effects and the broad band $\mathrm{Z} / \mathrm{n}$. Since the $\Delta p / p$ increases as a bunch approaches transition energy, it is necessary to take into account the momentum acceptance of the FMI. From these simulations we find that the $\gamma_{t}$-jump scheme is preferable compared to FFTC. However, for emittance $\leq 0.1 \mathrm{eVs}$, and with the fast ramps the benefits are limited. With the FFTC scheme the emittance growth will be in between those for NTPJ and the $\gamma_{t}$-jump scheme. For emittance $\geq 0.2 \mathrm{eVs}$ we find that the FFTC and $\gamma_{t}$-jump schemes give almost no emittance growth, while, with the NTPJ there is a max-
Table II

The results of the longitudinal beam dynamics simulations for transition crossing using ESME. The fractional growth $\Delta \epsilon / \epsilon$ for different schemes is listed.

\begin{tabular}{|l|l|l|l|r|}
\hline $\begin{array}{l}\left.\dot{\gamma}\right|_{\text {trans. }} \\
\left(\mathrm{sec}^{-1}\right)\end{array}$ & $\begin{array}{l}\text { Init. Long. } \\
\text { Emittance } \\
(\mathrm{eVs})\end{array}$ & NTPJ & FFTC & $\gamma_{t}$-jump \\
\hline 167 & 0.1 & 3.0 & $0.6^{a}$ & 0.15 \\
& 0.2 & 0.09 & $0.04^{a}$ & 0.02 \\
\hline 300 & 0.1 & 1.6 & - & 0.25 \\
& 0.2 & 0.06 & - & 0.02 \\
\hline
\end{tabular}

${ }^{a}$ In these cases the ESME simulations have been carried out for $\left.\dot{\gamma}\right|_{\text {transition }}=169 / \mathrm{sec}$.

Comparison between Gamma_t, FFTC and NTPJ Schemes For FMI Using ESME, $\epsilon_{1}$ (initial) $=0.1 \mathrm{eV}-\mathrm{sec}$

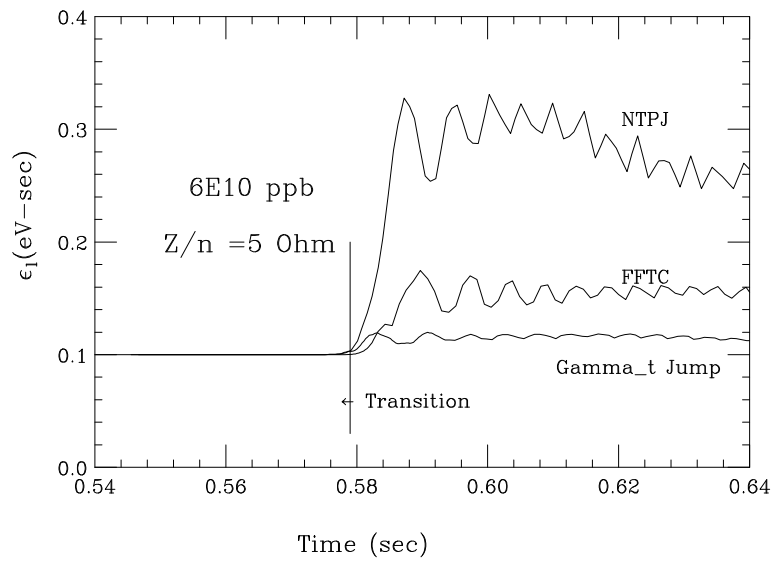

Figure 1. A comparison between $\gamma_{t}$-jump, FFTC and NTPJ schemes for the FMI. The initial emittance is $0.1 \mathrm{eVs}$, number of protons per bunch $=6 \times 10^{10}$. The $\left.\dot{\gamma}\right|_{\text {transition }}=167 / \mathrm{sec}$.

imum of about $10 \%$ emittance growth. Thus, with $\left.\dot{\gamma}\right|_{\text {transition }}$ $=300 / \mathrm{sec}$ and with $\epsilon_{l} \geq 0.2 \mathrm{eVs}$ we may not need any of the schemes like FFTC or the $\gamma_{t}$-jump for transition crossing in the FMI.

In a separate set of calculations we have estimated the negative mass instability using ESME. Our results confirm the calculations of $\mathrm{Ng}$ [6], who employed the analysis of Hardt[7]. We find for $6 \times 10^{10}$ protons/bunch a limit of $\epsilon_{l} \leq 0.16 \mathrm{eVs}$ for $\left.\dot{\gamma}\right|_{\text {transition }}=167 / \mathrm{sec}$ and $\epsilon_{l} \leq 0.12 \mathrm{eVs}$ for $\left.\dot{\gamma}\right|_{\text {transition }}=$ $300 / \mathrm{sec}$.

\section{SUMMARY AND CONCLUSIONS}

We have simulated the transition crossing for the proton beam with $6 \times 10^{10}$ particle /bunch. Three different schemes of transition crossing in the FMI have been investigated. We find that 
for an operating scenario of $\left.\dot{\gamma}\right|_{\text {transition }}=300 / \mathrm{sec}$ and $\epsilon_{l} \geq 0.2$ eVs we do not need any special schemes like $\gamma_{t}$-jump or FFTC.

Authors would like to acknowledge Dr. K.Y. Ng for useful discussions, especially the treatment of negative mass instability.

\section{References}

[1] J.A. Maclachlan, User's Guide to ESME v.8.13, Fermilab TM-1856(1994).

[2] D. Bogert, W. Fowler, S. Holmes, P. Martin and T. Pawlak, 'The status of the Fermilab Main Injector Project' (these proceedings).

[3] A. Sorenssen, Part. Accelerators. Vol. 6 (1975) 141.

[4] J. Griffin, Synchrotron Phase Transition Crossing Using an RF Harmonic, Fermilab TM 1734 (1991).

[5] E.D. Courant and H.S. Snyder, Annals of Phys. 3(1958) page 1 .

[6] I. Kourbanis and K.Y.Ng, Proc. Part. Accel. Conf. (1993) 3630 .

[7] W. Hardt, Proc. 9th Int. Conf. on High Energy Accelerators, Stanford 1974. 